\title{
Reviews of "Repurposed antiviral drugs for COVID- 19; interim WHO SOLIDARITY trial results"
}

\author{
Alberto Borobia' ${ }^{1}$, Antonio J Carcas ${ }^{2}$, Marie Warrer Munch ${ }^{3}$, \\ Stephen Shiboski ${ }^{4}$, Emily Walsh ${ }^{5}$ \\ ${ }^{1}$ La Paz University Hospital, Clinical Pharmacology, Spain, \\ ${ }^{2}$ Clinical Pharmacology Department. La Paz University Hospital. School of Medicine. Universidad \\ Autónoma de Madrid. IdiPAZ, \\ ${ }^{3}$ Rigshospitalet, Department of Intensive Care, Denmark, \\ ${ }^{4}$ Professor Emeritus, University of California San Francisco, Epidemiology and Biostatisics, USA, \\ ${ }^{5}$ Consultant, Kaiser Permanente, Care Management Institute, USA
}

Published on: Nov 11, 2020

DOI: $10.1162 / 2 \mathrm{e} 3983 f 5.3 a 75 \mathrm{cbb} 9$

License: Creative Commons Attribution 4.0 International License (CC-BY 4.0). 
To read the original manuscript, click the link above.

Summary of Reviews: This reliable study suggests four antivirals have no effect on important clinical outcomes in hospitalized COVID-19 patients. While the randomized control trial is thorough, reviewers note missing descriptions of study protocol, patient eligibility, \& adverse reactions.

\section{Reviewer 1 (Alberto Borobia, Antonio J Carcas) |}

Reviewer 2 (Marie Warrer Munch) | प्र०००

Reviewer 3 (Stephen Shiboski) | प्र०ण

Reviewer 4 (Emily Walsh)

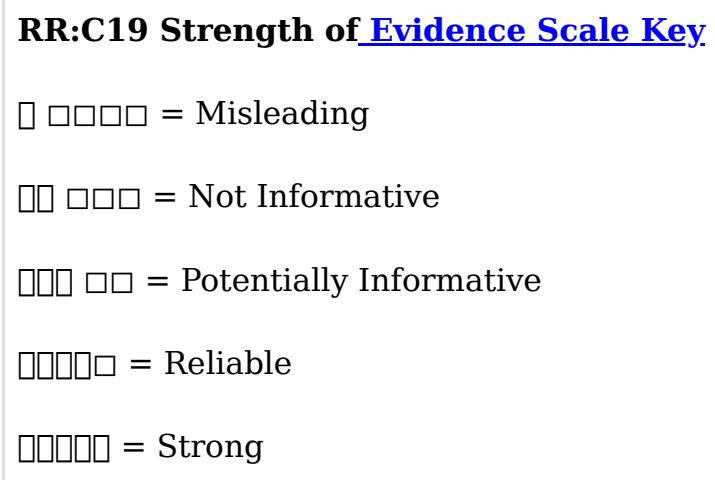

To read the reviews, click the links below. 